\title{
Identification of a mutated BHK-21 cell line that became less susceptible to Japanese encephalitis virus infection
}

\author{
Tianbing Ding ${ }^{1 \dagger}$, Wei Zhang ${ }^{2 \dagger}$, Wenyu $\mathrm{Ma}^{1}$, Junping Ren ${ }^{{ }^{*}}$
}

\begin{abstract}
The pathogenesis of Japanese encephalitis virus (JEV) is not definitely elucidated as the initial interaction between virus and host cell receptors required for JEV infection is not clearly defined yet. Here, in order to discover those membrane proteins that may be involved in JEV attachment to or entry into virus permissive BHK-21 cells, a chemically mutated cell line (designated 3A10-3F) that became less susceptible to JEV infection was preliminarily established and selected by repeated low moi JEV challenges and RT-PCR detection for viral RNA E gene fragment. The susceptibility to JEV of 3A10-3F cells was significantly weakened compared with parental BHK-21 cells, verified by indirect immunofluorescence assay, virus plague formation assay, and flow cytometry. Finally, two-dimensional electrophoresis (2-DE) coupled with LC-MS/MS was utilized to recognize the most differentially expressed proteins from membrane protein extracts of 3A10-3F and BHK-21 cells respectively. The noted discrepancy of membrane proteins included calcium binding proteins (annexin A1, annexin A2), and voltage-dependent anion channels proteins (VDAC 1, VDAC 2), suggesting that these molecules may affect JEV attachment to and/or entry into BHK21 cells and worthy of further investigation.
\end{abstract}

\section{Findings}

Japanese encephalitis virus (JEV), a member of genus Flavivirus in the family Flaviviridae, is the causative agent of Japanese encephalitis (JE), the mosquito-borne viral encephalitis epidemic in eastern, southeastern and southern Asia, leading to an estimated $\sim 50,000$ infections annually, of which $\sim 15,000$ will die and up to $50 \%$ of survivors are left with severe residual neurological complications $[1,2]$. During the past decades, JE is spreading beyond its traditional boundaries and has reported from previously unaffected areas such as Saipan islands, Pakistan and northern Australia [3-5]. Coupled with a high rate of mortality and residual neurological complications in survivors, it makes JE a serious public health problem in tropical and subtropical areas in the world.

The first step of virus infection requires the interaction between virus attachment proteins (VAPs) and cellular

\footnotetext{
* Correspondence: juren@utmb.edu

† Contributed equally

'Department of Microbiology, the Fourth Military Medical University, Xi'an,

Shaanxi, 710032, PR China

Full list of author information is available at the end of the article
}

receptors, which is known to contribute to host range, tissue tropism and viral pathogenesis. In the cases of flaviviruses including JEV, envelope glycoprotein E, protruding as spikes on the surface of virions, is considered to be the dominant antigen in mediating receptor binding and membrane fusion, hemagglutination, neutralization and virulence [6-8]. As of enzootic nature, JEV maintains a natural cycle among birds, pigs, and other vertebrate hosts by mosquitoes without serious sickness, and thus the cells from above species, such as African green monkey kidney cells (Vero), baby hamster kidney cells (BHK21), and Aedes albopictus cells (C6/36) [9], are frequently applied in studies associated with JEV pathogenicity due to their ability to permit JEV entry and replication within them. Such broad tropism of JEV rationally suggests that most possibly there exists more than one cellular receptor responsible for virus binding and entry into susceptible cells above mentioned.

To date, little is known about JEV cellular receptors. Much earlier, a report stated that a $74 \mathrm{kDa}$ protein on Vero cells was found to be capable of binding JEV and might be involved in virus uptake process [10]. A recent paper also indicated that several proteins on the surface 
of C6/36 cells with masses ranging from $35-80 \mathrm{kDa}$ and 150-200 kDa may bind to JEV, but failed to identify specific proteins by mass spectroscopic fingerprint analysis [11]. In 2009, a paper reported that heat shock protein 70 is a putative receptor for JEV on mouse neuroblastoma (Neuro2a) cells [12]. Hence, the detailed interaction between JEV and its putative receptor(s) is not exclusively defined yet.

One of the most convincing methods to verify a putative virus receptor is to transfer the receptor gene into a cell line that cannot bind virus and later demonstrate that the receptor-negative cell acquires the ability to bind virus and permit virus to replicate within it after the receptor gene is regained [13]. For this reason, the availability of a specific virus receptor-negative/-defective cell line is usually a prerequisite for virus receptor confirmation. Unfortunately, such JEV receptor-negative/-defective cell line is not currently available after our extensive searching for animal and human cell lines, and thus it has to be established artificially.

The successful identification of the cellular receptor for anthrax toxin provided us a practical strategy to create any specific virus receptor-negative/-defective cells [14]. Here, BHK-21 cells, permissive to JEV entry and replication, were subject to co-culture with a DNA alkylating mutagen ICR-191 to introduce random small DNA deletions and frame shift mutations in the genes of normally cultured BHK-21 cells under conditions that led to $\sim 90 \%$ cell death. The survived cells were grown up but underwent a slow die-off for 4 weeks. These viable but mutated BHK-21 cells were successively subject to several rounds of infectious JEV challenge at a low moi of 0.1 , subcloned by limiting dilution, and then each individual subclones was detected by RT-PCR for negative JEV $E$ gene RNA fragment. RT-PCR primers were synthesized according to the RNA sequence of JEV SA-14 strain [15] from 1528 to 2234 of $E$ gene conserved fragment, which were sense-strand primer, 5'-CGGAATTCGAGAAGTCACACTGGACTGTGA GCC-3', and antisense-strand primer, 5'-CGCTGC CAGTC TTTGAGCTCCCTTCAAAGT-3'. Finally, such a JEV RNA-negative mutated cell line, designated 3A10$3 \mathrm{~F}$, was picked out after two cycles of limiting dilution cloning from eight subclones of mutated BHK-21 cells (Figure 1). Further, the susceptibility of 3A10-3F to JEV infection was tested by indirect immunofluorescence assay (IFA), viral plaque formation assay, and flow cytometry. In IFA, JEV E protein synthesized within the cells was detected by monoclonal antibody against $\mathrm{E}$ protein. The results clearly displayed that fluorescent signals from 3A10-3F were much weaker (Figure $2 \mathrm{~b}$ ) than BHK-21 cells (Figure 2d) with the same dosage of JEV, while both negative controls of the two cell lines appeared the same (Figure 2a and Figure 2c).

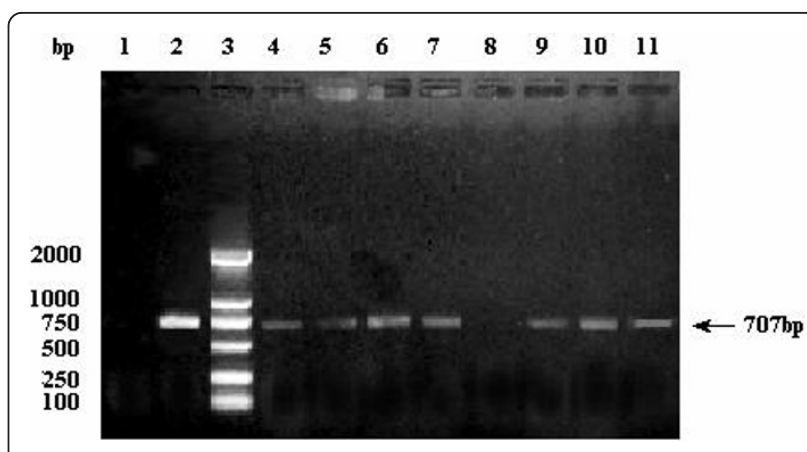

Figure 1 Selection of mutated cell lines negative of JEV E gene RNA fragments by RT-PCR. Eight single-cell clones of mutated cells (lanes 4-11) were detected in triplicate. The arrow indicates 707 bp fragments containing the conserved JEV E gene sequence. Only one single-cell clone, designated 3A10-3F, showed JEV RNA negative (lane 8). BHK-21 cells (lane 1) and BHK-21 cells infected with JEV (lane 2) were negative and positive controls respectively. Lane 3 was DL-2000 DNA marker.

To reckon the susceptibility of $3 \mathrm{~A} 10-3 \mathrm{~F}$ to JEV, virus plaque formation assay and flow cytometry were applied. It appeared that JEV was able to replicate in the same kinetic mode in either $3 \mathrm{~A} 10-3 \mathrm{~F}$ or BHK-21 cells at 0-48 h post-infection (Figure 3 ), but the numbers of infectious virions detected by plaque formation assay in $3 \mathrm{~A} 10-3 \mathrm{~F}$ cells were remarkably declined. At moi of 1 , the highest JEV titer at $48 \mathrm{~h}$ post-infection from 3A10-3F cells was decreased by 2 orders of logarithm compared with that of titer from BHK-21 cells (Figure 3a). At moi of 10 , the highest titer from 3A10-3F cells was lowered by 1 order of logarithm compared with that of from BHK-21 cells. Conspicuously there was a similar decline mode of virus replication in 3A10-3F cells compared with its parental cells between the two moi of JEV.

In flow cytometry, the binding between 3A10-3F cells and JEV was $2.10 \%$ (Figure $4 \mathrm{a}$ ), much lower than the binding between BHK-21 cells and JEV (48.84\%, Figure 4b), clearly indicating that $3 \mathrm{~A} 10-3 \mathrm{~F}$ cells are defective of JEV binding because of the compromise of normal surface protein functioning for JEV attachment and/or entry. Besides, 3A10-3F cells appear morphologically similar (Figure 5a) to normal BHK-21 cells (Figure 5c), and still susceptible to HSV-1 (SM64 strain) infection as they manifested the similar CPEs (Figure 5b) as rapidly as BHK-21 cells did (Figure $5 \mathrm{~d}$ ). The reason to choose HSV-1 is that HSV binds to its surface protein molecules definitely different from that of JEV on BHK-21 cells, thus excluding the possibility that several mosquito-borne flaviviruses (such as JEV, Dengue virus, West Nile virus, etc) may attach the same protein molecules when virus-host interaction occurs [16]. Therefore, the HSV-1 infection test reinforced that resistance of $3 \mathrm{~A} 10-3 \mathrm{~F}$ cells to JEV infection was specific for JEV (machinery for gene expression appeared 

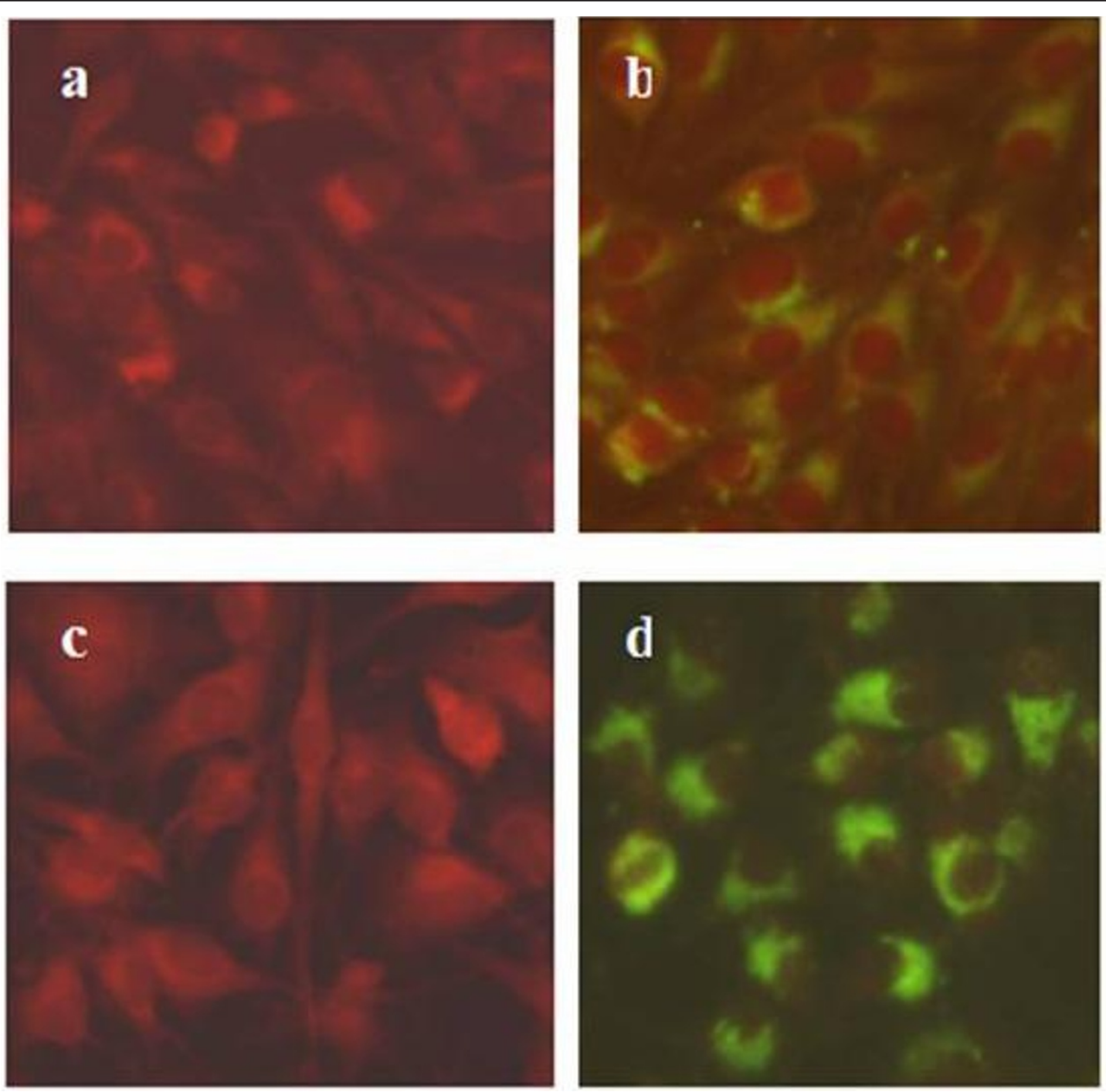

Figure 2 JEV binding to 3A10-3F and BHK-21 cells measured by indirect immunofluorescence assay (IFA). JEV antigen were detected with anti-JEV mAb and FITC-conjugated goat anti-mouse lgG at $24 \mathrm{hr}$ post-infection, and uninfected 3A10-3F cells (a) and BHK-21 cells (c) were negative controls. The cytoplasm and nuclei were stained red by Evans blue, and green fluorescent signals of viral antigen were significantly weaker in 3A10-3F cells (b) than in BHK-21 cells (d) (Magnification: $\times 400)$.

intact), and was much less probably resulted from the decreased virus replication within the cells but from less virus binding to cell surface molecules necessary for JEV attachment and/or entry.

All data above implied that (i) JEV was still permissive to replicate within 3A10-3F cells but at a low level; (ii) JEV might enter 3A10-3F cells through different molecule routes, possibly high affinity and low affinity receptors [10]; and (iii) 3A10-3F cells became resistant to JEV infection most likely due to the altered surface protein expression after chemical mutagenesis rather than the mutations of certain intracellular factors essential for JEV replication.

Finally, two-dimensional electrophoresis (2-DE) coupled with mass spectrometry was used to determine those differentially expressed proteins between $3 \mathrm{~A} 10-3 \mathrm{~F}$ and BHK-21 cell membrane protein extracts following Mirza's method [17]. Altogether 23 spots of differentially expressed proteins on SDS-PAGE gels were picked out (Figure 6) upon computerized sifting criteria (the smooth parameter was set to a value of 2; the minimum area was above 8 pixels; the saliency parameter was experimentally adjusted to 1$)$, including six up-regulated spots $(11,12,14$, $17,19,21)$ and six down-regulated spots $(1,4-7,9)$ in 3A10-3F cells compared with BHK-21 cells, seven spots $(13,15,16,18,20,22,23)$ solely expressed in 3A10-3F cells and four spots $(2,3,8,10)$ in BHK-21 cells respectively. These spots were subject to mass spectrometry analysis in combination with a mouse peptide database 


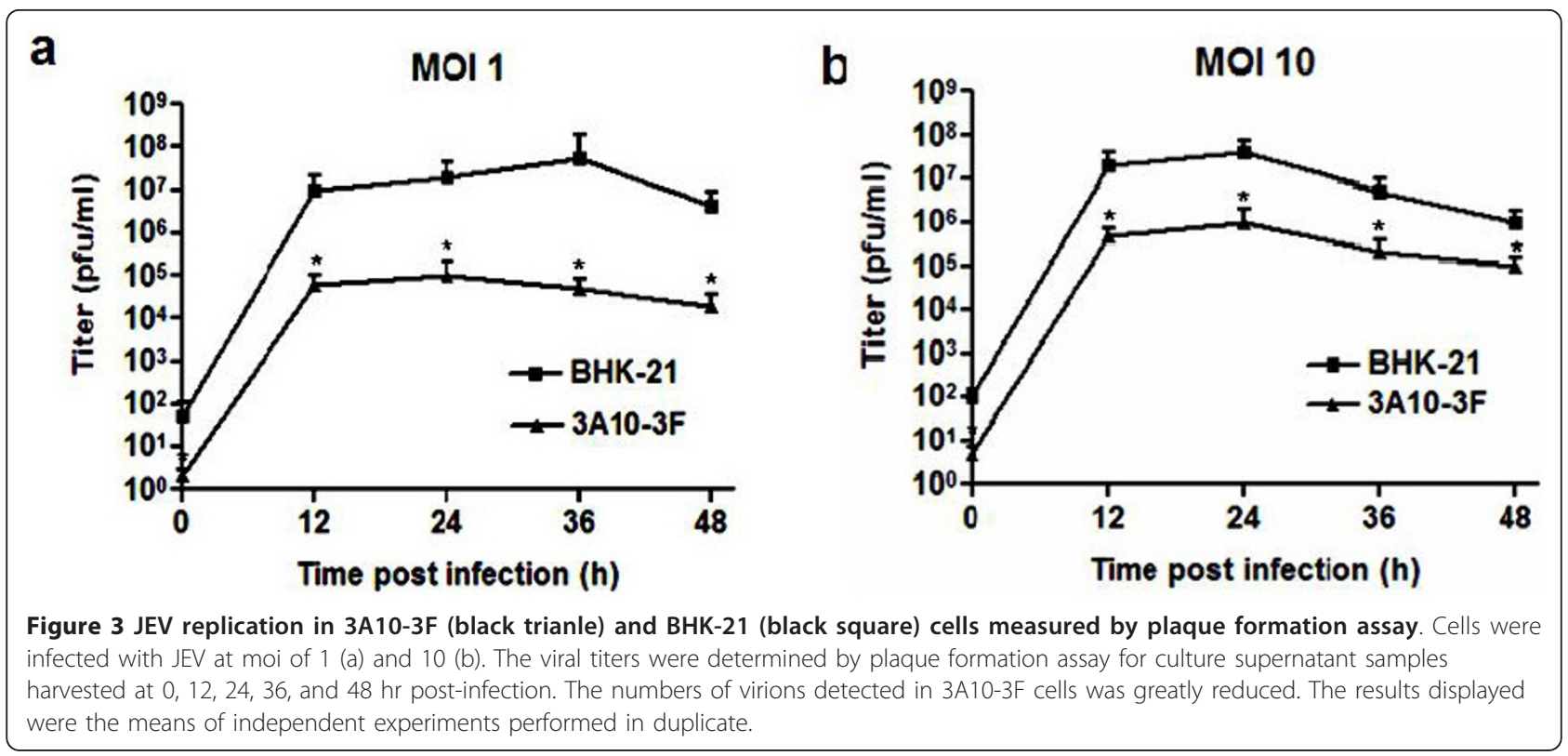

(NCBInr) searching, and the results indicated that 14 spots among the 23 samples (spots 1, 3-5, 7, 10-12, 14-16, 20, 22 , and 23) were determined with good peptide coverage and significant scoring (Additional file 1 table S1). Not surprisingly, not all proteins extracted were membrane proteins, but all proteins with most altered abundance must have been disclosed. With such certitude, four membrane proteins from 3A10-3F cells recognized by mass spectrometry, annexin 1 and annexin 2, voltage-dependent anion channel (VDACs) 1 and 2, were of our particular interests.
Annexins are a family of structurally related proteins whose common properties are to bind both phospholipids and cellular membranes in a calcium-dependent manner [18]. Annexin 1 involves in diverse cellular roles, including membrane fusion, exocytosis, differentiation, apoptosis, calcium channels, inflammatory reactions, and interaction with cytoskeletal proteins [19]. To date, few but one report [20] referred the role of annexin 1 in viral infection, stating that infectious pancreatic necrosis virus (IPNV) infection to fish cell increased the expression of salmon annexin 1 , and the
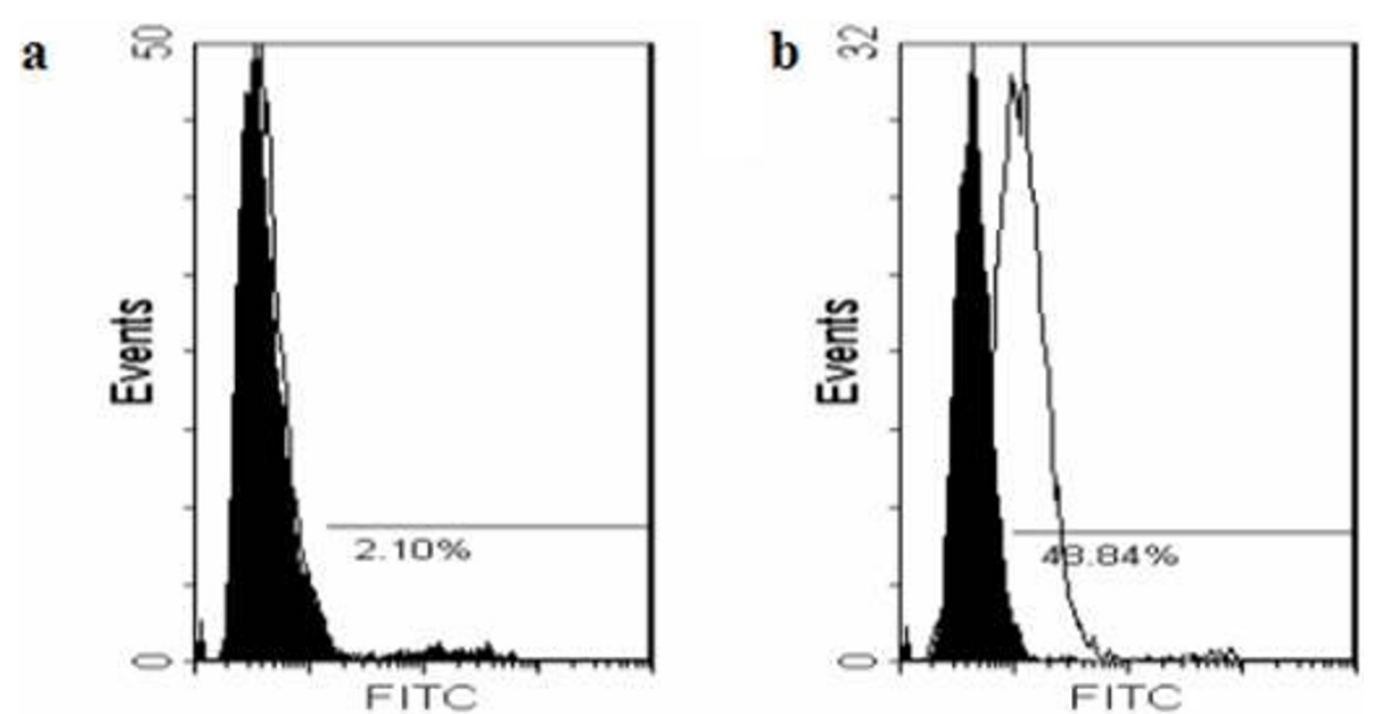

Figure 4 JEV binding to 3A10-3F and BHK-21 cells measured by flow cytometry. Cells were incubated with JEV for 1 hr and were stained with rabbit anti-JEV antibodies followed by FITC-conjugated goat anti-rabbit IgG. Limited binding of JEV to 3A10-3F cells was shown (2.10\%, a), whereas JEV significantly bound BHK-21 cells $(48.84 \%, b)$. 

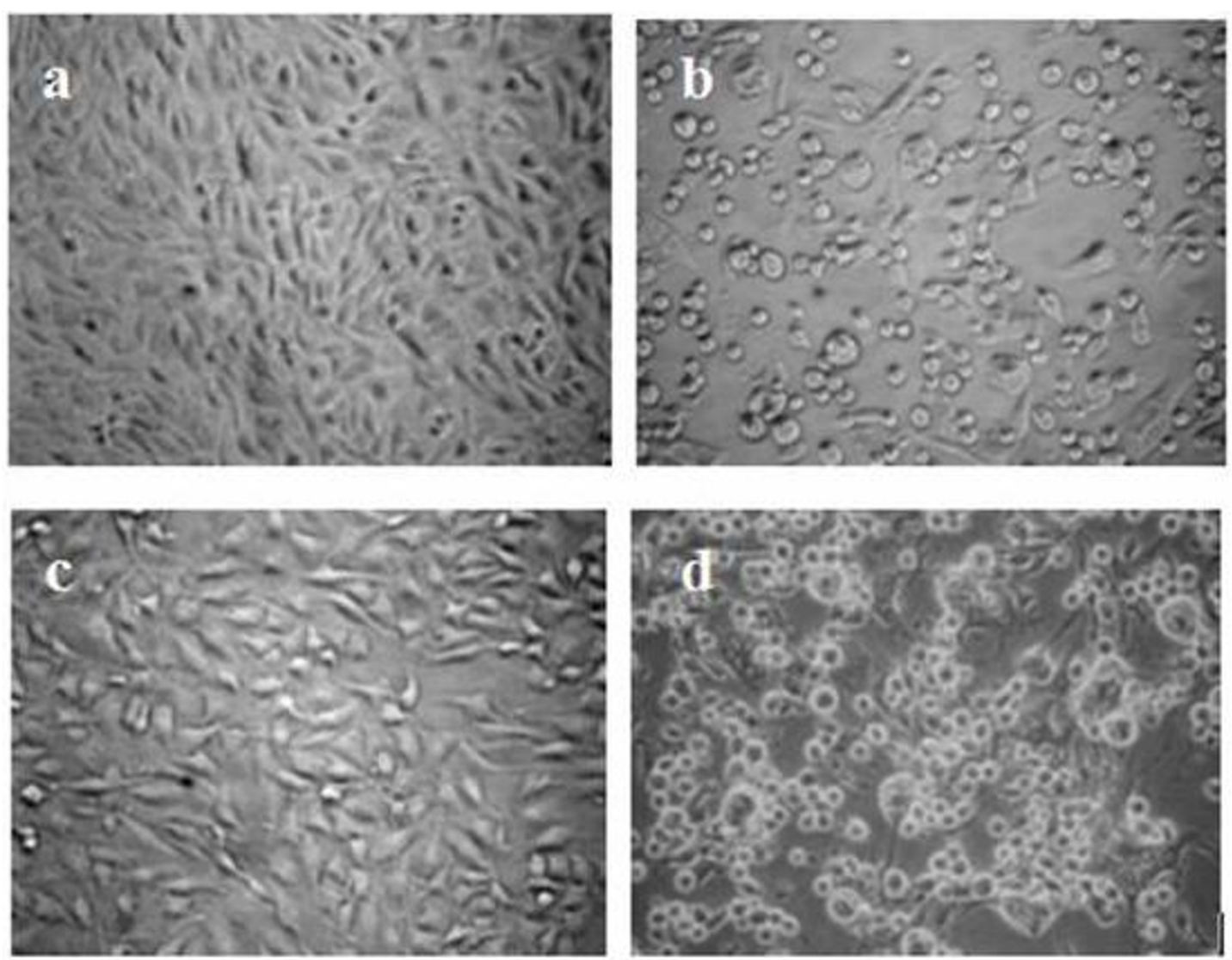

Figure 5 Cell morphology and CPEs of 3A10-3F and BHK-21 cells under a phase-contrast microscope. 3A10-3F cells (a) appeared morphologically similar to parental BHK-21 cells (c). After infected with HSV-1, 3A10-3F cells (b) appeared similar CPEs to normal BHK-21 cells infected with HSV-1(d) (Magnification: $\times 200$ ).

increased expression of salmon annexin 1 inhibited the apoptosis of IPNV-infected cells and supported the growth of IPNV in cells. In addition, annexin 2 had been identified as a receptor for cytomegalovirus (CMV) and respiratory syncytial virus (RSV) [21,22], and also promoted entry of HIV-1 into cells and proper assembly of HIV-1 in cells [23,24]. In our study, the expression of annexin 1 and annexin 2 were found to be significantly reduced on 3A10-3F cells, possibly suggesting that they may be involved in JEV attachment and/or entry.

VDACs is a multigene family of evolutionarily conserved and well characterized porins found in outer mitochondrial membranes of all eukaryotes [25], where they control homeostasis by transport of ATP and ADP [26]. Numerous research groups also reported the presence of VDAC proteins in the plasma membrane of various cell types [27,28]. VDAC 1 contributed to ATP transport across the plasma membrane of murine cells [29], and could act as NADH-ferricyanide reductase to inhibit the release of synthesized ATP and greatly decrease the activity of exogenous NADH/cytochrome-c system of intact mitochondria [30], however the significance of its total absence from 3A10-3F on JEV infection is not reported. Mammalian VDAC 2 exhibited other more biological activities, such as interaction with $\mathrm{Bcl}-2$ family proteins, critical regulators of apoptosis [31]. In our study, VDAC 2 should not be a candidate for JEV binding molecule as it displayed an increased expression (16-fold) on 3A10-3F cells.

Nevertheless, some membrane molecules other than annexins and VDACs may not be excluded to mediate JEV entry into cells. It is not unusual that a virus particle utilizes multiple surface proteins during cell entry [32-34]. Several viruses utilize at least two different molecules to interact with their host cells via: (i) the binding receptors, merely serve as attachment factors that concentrate or recruit viruses on cell surface; (ii) co-receptors, that are used by the virus after binding to the cells, not only bind viruses but are also responsible for directing the bound viruses into endocytic pathways and for transmitting followed signals to the cytoplasm [35].

Taken together, the mutated cell line 3F10-3F from parental BHK-21 cells became less susceptible to JEV 


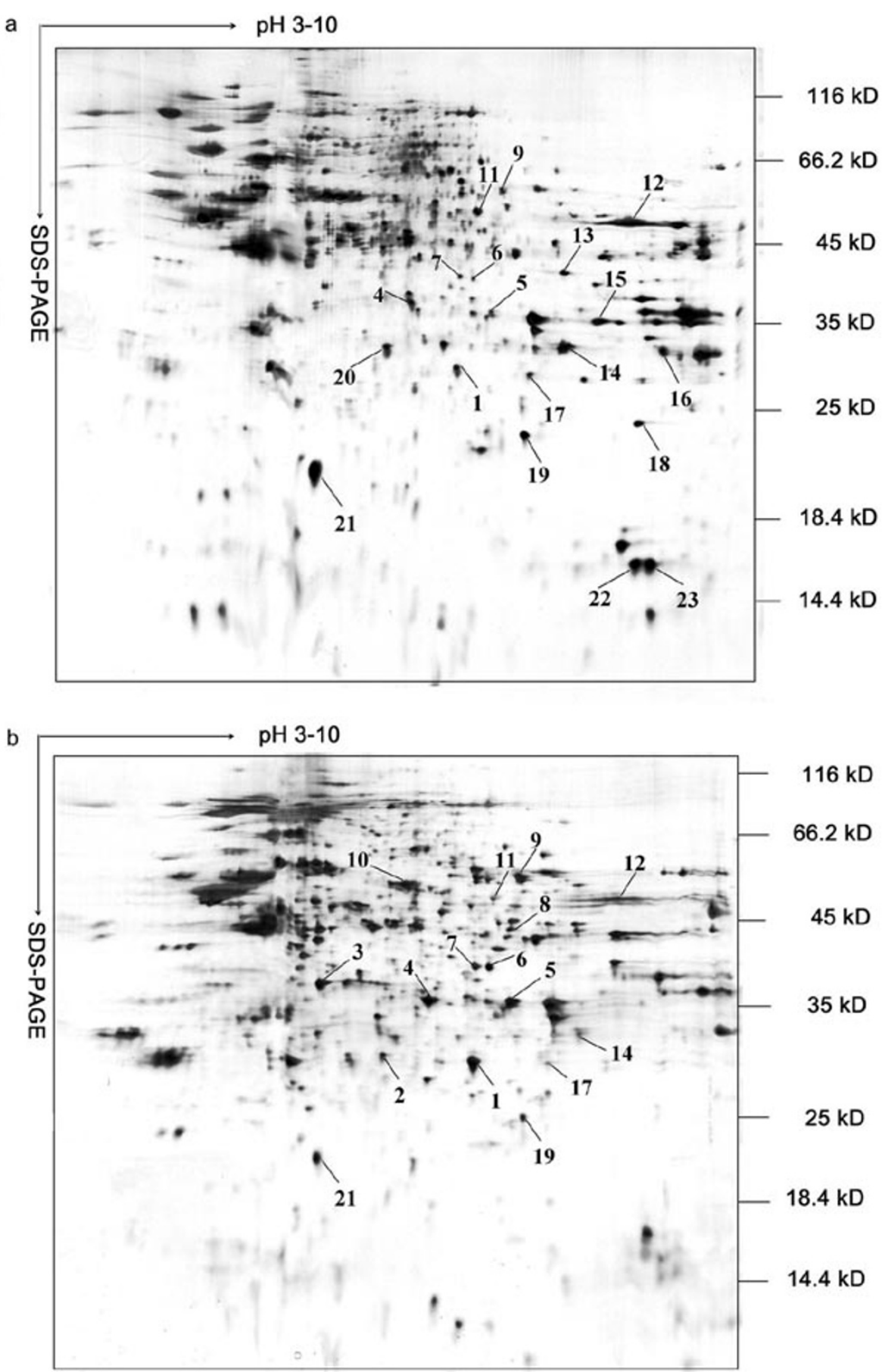

Figure 6 Representative 2-DE maps of 3A10-3F and BHK-21 cell membrane protein extracts. Membrane proteins from 3A10-3F (a) and BHK-21 cells (b) were separated by 2-DE and stained with sliver. 23 most differentially expressed protein spots were marked with arrows, and these spots were analyzed by LC-MS/MS. Three separate gels were prepared for each cell line.

infection, and the discrepancy of membrane proteins between daughter and parental cells thus provides important clues to further investigate the individual role of the membrane proteins in JEV infection on BHK cells.

\section{Additional material}

Additional file 1: Table 1: Comparison of LC-MS/MS recognized proteins between $3 \mathrm{~A} 10-3 \mathrm{~F}$ and BHK-21 cells. A wider table describing recognized protein properties in MS Word .doc format. 


\section{List of abbreviations}

BHK: baby hamster kidney; CPE: cytopathic effect; FITC: fluorescein isothiocyanate; HSV: herpes simplex virus; IFA: indirect immunofluorescence assay; ICR-191: (6-chloro-9-[3-(2-chloroethylamino)propylamino]-2methoxyacridine; JEV: Japanese encephalitis virus; LC-MS: liquid chromatography-mass spectrometry; moi: multiplicity of infection; VDAC: voltage-dependent anion channels protein

\section{Acknowledgements}

The authors would especially like to thank Dr. Yusong Ruan, State Key Laboratory of Biomembrane and Membrane Biotechnology, Institute of Zoology, Chinese Academy of Sciences, for technical support for 2-DE and LC-MS/MS analysis. This work is supported by Natural Science Foundation of China (30600526, 30470091).

\section{Author details}

'Department of Microbiology, the Fourth Military Medical University, Xi'an, Shaanxi, 710032, PR China. ${ }^{2}$ Department of Biopharmaceutics, School of Pharmacy, the Fourth Military Medical University, Xi'an, Shaanxi, 710032, PR China.

\section{Authors' contributions}

TD and JR conceived the study and drafted the manuscript. TD carried out the chemical mutation on BHK-21 cells. WZ did RT-PCR. JR carried out IFA, plaque formation assay, flow cytometry, and 2-DE. TD and JR did bioinformatic analysis. WM and JR proofread the manuscript. All authors read and approved the final manuscript.

\section{Competing interests}

The authors declare that they have no competing interests.

Received: 23 October 2010 Accepted: 14 March 2011

Published: 14 March 2011

\section{References}

1. Tsai TF: New initiatives for the control of Japanese encephalitis by vaccination: minutes of a WHO/CVI meeting, Bangkok, Thailand, 13-15 October 1998. Vaccine 2000, 18(Suppl 2):1-25.

2. Solomon T: Control of Japanese encephalitis - within our grasp? New Eng J Med 2006, 355:869-871.

3. Okada SF, O'Neal WK, Huang P, Nicholas RA, Ostrowski LE, Craigen WJ, Paul WS, Moore PS, Karabatsos N, Flood SP, Yamada S, Jackson T, Tsai TF: Outbreak of Japanese encephalitis on the island of Saipan. J Infect Dis 1993, 167:1053-1058.

4. Igarashi A, Tanaka M, Morita K, Takasu T, Ahmed A, Ahmed A, Akram DS, Waqar MA: Detection of West Nile and Japanese encephalitis viral genome sequences in cerebrospinal fluid from acute encephalitis cases in Karachi, Pakistan. Microbiol Immunol 1994, 38:827-830.

5. Hanna JN, Ritchie SA, Phillips DA, Lee JM, Hills SL, van den Hurk AF, Pyke AT, Johansen CA, Mackenzie JS: Japanese encephalitis in north Queensland, Australia, 1998. Med J Austra 1999, 170:533-536.

6. Heinz FX: Epitope mapping of flavivirus glycoproteins. Adv Virus Res 1986, 31:103-168.

7. McMinn PC: The molecular basis of virulence of the encephalitogenic flaviviruses. J Gen Virol 1997, 78:2711-2722.

8. Wu KP, Wu CW, Tsao YP, Kuo TW, Lou YC, Lin CW, Wu SC, Cheng JW: Structural basis of a flavivirus recognized by its neutralizing antibody: solution structure of the domain III of Japanese encephalitis virus envelope protein. J Biol Chem 2003, 278:46007-46013.

9. Mackenzie JS, Gubler DJ, Petersen LR: Emerging flaviviruses: the spread and resurgence of Japanese encephalitis, West Nile and dengue viruses. Nat Med 2004, 10(12 Suppl):S98-109.

10. Kimura T, Kimura-Kuroda J, Nagashima K, Yasui K: Analysis of virus-cell binding characteristics on the determination of Japanese encephalitis virus susceptibility. Arch Virol 1994, 139:239-251.

11. Boonsanay V, Smith DR: Entry into and production of the Japanese encephalitis virus from C6/36 cells. Intervirology 2007, 50:85-92.

12. Das S, Laxminarayana SV, Chandra N, Ravi V, Desai A: Heat shock protein 70 on Neuro2a cells is a putative receptor for Japanese encephalitis virus. Virology 2009, 385:47-57.
13. Haywood AM: Virus receptors: binding, adhesion strengthening, and changes in viral structure. J Virol 1994, 68:1-5.

14. Bradley KA, Mogridge J, Mourez M, Collier RJ, Young JA: Identification of the cellular receptor for anthrax toxin. Nature 2001, 414(6860):225-229.

15. Nitayaphan S, Grant JA, Chang GJ, Trent DW: Nucleotide sequence of the virulent SA-14 strain of Japanese encephalitis virus and its attenuated vaccine derivative, SA-14-14-2. Virology 1990, 177:541-552.

16. Ren JP, Ding TB, Zhang W, Song JH, Ma WY: Does Japanese encephalitis virus share the same cellular receptor with other mosquito-borne flaviviruses on the C6/36 mosquito cells? Virol J 2007, 6:83.

17. Mirza SP, Halligan BD, Greene AS, Olivier M: Improved method for the analysis of membrane proteins by mass spectrometry. Physiol Genomics 2007, 30:89-94

18. Gerke V, Moss SE: Annexins: from structure to function. Physiol Rev 2002, 82:331-371.

19. Flower RJ, Rothwell NJ: Lipocortin-1: cellular mechanisms and clinical relevance. Trends Pharmacol Sci 1994, 15:71-76.

20. Hwang HJ, Moon CH, Kim HG, Kim JY, Lee JM, Park JW, Chung DK: Identification and functional analysis of salmon annexin 1 induced by a virus infection in a fish cell line. J Virol 2007, 81:13816-13824.

21. Raynor CM, Wright JF, Waisman DM, Pryzdial EL: Annexin II enhances cytomegalovirus binding and fusion to phospholipids membranes. Biochem 1999, 38:5089-5095.

22. Malhotra R, Ward M, Bright H, Priest R, Foster MR, Hurle M, Blair E, Bird M: Isolation and characterization of potential respiratory syncytial virus receptor(s) on epithelial cells. Microbes Infect 2003, 5:123-133.

23. Ma G, Greenwell-Wild T, Lei K, Jin W, Swisher J, Hardegen N, Wild CT, Wahl SM: Secretory leukocyte protease inhibitor binds to annexin II, a cofactor for macrophage HIV-1 infection. J Exp Med 2004, 200:1337-1346.

24. Ryzhova EV, Vos RM, Albright AV, Harrist AV, Harvey T, Gonzalez-Scarano F: Annexin 2: a novel human immunodeficiency virus type $1 \mathrm{Gag}$ binding protein involved in replication in monocyte-derived macrophages. J Virol 2006, 80:2694-2704.

25. Hodge T, Colombini M: Regulation of metabolite flux through voltagegating of VDAC channels. J Membr Biol 1997, 157:271-279.

26. Rostovtseva T, Colombini M: VDAC channels mediate and gate the flow of ATP: implications for the regulation of mitochondrial function. Biophys J 1997, 72:1954-1962

27. Yu WH, Forte M: Is there VDAC in cell compartments other than the mitochondria? J Bioenerg Biomembr 1996, 28:93-100.

28. Yu WH, Wolfgang W, Forte M: Subcellular localization of human voltagedependent anion channel isoforms. J Biol Chem 1995, 270:13998-14006.

29. Okada SF, O'Neal WK, Huang P, Nicholas RA, Ostrowski LE, Craigen WJ Lazarowski ER, Boucher RC: Voltage-dependent anion channel-1 (VDAC-1) contributes to ATP release and cell volume regulation in murine cells. J Gen Physiol 2004, 124:513-526.

30. Baker MA, Lane DJ, Ly JD, De Pinto V, Lawen A: VDAC1 is a transplasma membrane NADH-ferricyanide reductase. J Biol Chem 2004, 279:4811-4819.

31. Vander Heiden MG, Li XX, Gottleib E, Hill RB, Thompson CB, Colombini M: $\mathrm{BCl}-\mathrm{xL}$ promotes the open configuration of the voltage-dependent anion channel and metabolite passage through the outer mitochondrial membrane. J Biol Chem 2001, 276:19414-19419.

32. Sieczkarski SB, Whittaker GR: Viral entry. Curr Top Microbiol Immunol 2004, 285:1-23.

33. Nishimura Y, Shimojima M, Tano Y, Miyamura T, Wakita T, Shimizu H: Human P-selectin glycoprotein ligand-1 is a functional receptor for enterovirus 71. Nat Med 2009, 15:794-797.

34. Yamayoshi S, Yamashita Y, Li J, Hanagata N, Minowa T, Takemura T, Koike S: Scavenger receptor B2 is a cellular receptor for enterovirus 71. Nat Med 2009, 15:798-801.

35. Smith AE, Helenius A: How viruses entry animal cells. Science 2004 304(5668):237-242

doi:10.1186/1743-422X-8-115

Cite this article as: Ding et al:: Identification of a mutated BHK-21 cell line that became less susceptible to Japanese encephalitis virus infection. Virology Journal 2011 8:115. 